\title{
THE METHOD OF FUNDAMENTAL SOLUTIONS FOR STATIONARY HEAT CONDUCTION PROBLEMS IN ROTATIONALLY SYMMETRIC DOMAINS*
}

\author{
YIORGOS-SOKRATIS SMYRLIS ${ }^{\dagger}$ AND ANDREAS KARAGEORGHIS ${ }^{\dagger}$
}

\begin{abstract}
We propose an efficient boundary collocation method for the solution of certain two- and three-dimensional problems of steady-state heat conduction in isotropic bimaterials. In particular, in two dimensions we consider the case where a circular region composed of one material is coated with an annular region of another material. In three dimensions, we examine the corresponding case for axisymmetric domains. The proposed method involves the use of a domain decomposition technique in conjunction with a matrix decomposition algorithm. The circulant structure of the matrices appearing in this method is exploited by using fast Fourier transforms. The method is tested numerically on several problems.
\end{abstract}

Key words. heat conduction in layered materials, method of fundamental solutions, Laplace equation, axisymmetric domains

AMS subject classifications. Primary 35J25, 65N12, 65N38; Secondary 65N15, 65T50

DOI. $10.1137 / 040615213$

1. Introduction. We consider steady-state heat conduction in an isotropic bimaterial. The bimaterial is decomposed into two subdomains $\mathfrak{O}_{1}$ and $\mathfrak{O}_{2}$, with boundaries $\partial \mathfrak{O}_{1}$ and $\partial \mathfrak{O}_{2}$, respectively, which are separated by an interface $\mathcal{S}=\partial \mathfrak{O}_{1}$. The two materials in $\mathfrak{O}_{1}$ and $\mathfrak{O}_{2}$ are characterized by the thermal conductivity constants $k_{1}$ and $k_{2}$, respectively. The temperature distribution in each subdomain satisfies the Laplace equation. On the interface $\mathcal{S}$ we have continuity of the temperature

$$
u_{1}=u_{2}
$$

and continuity of the flux

$$
k_{1} \frac{\partial u_{1}}{\partial n}=k_{2} \frac{\partial u_{2}}{\partial n}
$$

where $\partial / \partial n$ denotes the normal derivative in the outward direction to $\mathfrak{O}_{1}$ on the interface. Examples and details about the modeling of such problems may be found in $[18,24]$.

The aim of this study is to develop efficient algorithms for the numerical solution of such problems in the case when $\overline{\mathfrak{D}}_{1} \subset \mathfrak{O}_{2}$ and both domains are axisymmetric about the same axis. In two dimensions this means that we consider the case where one material occupies a circular region and another material occupies a concentric annular region surrounding it. In three dimensions, we examine the case where one material occupies an axisymmetric region and is coated by another material occupying a region which is axisymmetric about the same axis.

\footnotetext{
${ }^{*}$ Received by the editors September 16, 2004; accepted for publication (in revised form) May 24, 2005; published electronically January 27, 2006.

http://www.siam.org/journals/sisc/27-4/61521.html

${ }^{\dagger}$ Department of Mathematics and Statistics, University of Cyprus, P.O. Box 20537, 1678 Nicosia, Cyprus (smyrlis@ucy.ac.cy, andreask@ucy.ac.cy). This work was supported by University of Cyprus grant 8037-3/312-21005 21014. Part of the second author's work was undertaken while the author was a visiting professor in the Department of Mathematical and Computer Sciences, Colorado School of Mines, Golden, CO 80401.
} 
The numerical method that we use is the method of fundamental solutions (MFS) which is a boundary collocation meshless technique. In recent years, the MFS has become very popular, primarily because of the ease with which it can be implemented. It belongs to the family of Trefftz-type methods (see [4]), and details about its implementation and its various applications can be found in the survey articles $[6,7,11]$ and the books $[10,14]$.

We use a domain decomposition approach and a matrix decomposition algorithm. The domain is decomposed in a natural way into two subdomains, each containing a different material. Domain decomposition MFS applications can be found in [1], where heat conduction in layered materials is considered, and in [2, 9], where linear elasticity problems are studied. In the current work, in both the two- and the three-dimensional cases, the MFS discretization leads to the solution of linear systems whose coefficient matrices have block circulant structures which can be exploited by using fast Fourier transforms (FFTs). The circulant and/or block circulant structure of the matrices appearing when the MFS is applied to axisymmetric problems has been exploited in $[8,22]$. The idea of exploiting the FFTs to solve axisymmetric integral equations was introduced in [12]; see also [16]. The circulant structure of the coefficient matrices also arises from the application of other boundary methods such as the boundary element method (BEM), and this has been exploited in the past (see, e.g., [3, 15]). Details of applying a multiregion BEM approach to heat conduction problems in layered materials are presented in [19].

This paper is organized as follows. In section 2, we present the MFS formulation in the two-dimensional case. In section 3, we present the MFS formulation in the three-dimensional case. In section 4, we test numerically the proposed algorithms. Finally, in section 5, we give some concluding remarks.

\section{The two-dimensional case.}

2.1. The problem. We consider the bimaterial problem in $\mathbb{R}^{2}$,

$$
\begin{array}{ll}
\Delta u_{1}=0 & \text { in } \quad B_{\varrho_{1},} \\
\Delta u_{2}=0 & \text { in } \quad A_{\varrho_{1}, \varrho_{2}},
\end{array}
$$

subject to the boundary condition

$$
u_{2}=f \quad \text { on } \quad S_{\varrho_{2}}
$$

and the interface conditions

$$
\begin{aligned}
u_{1} & =u_{2} \quad \text { and } \\
k_{1} \frac{\partial u_{1}}{\partial n} & =k_{2} \frac{\partial u_{2}}{\partial n} \quad \text { on } \quad S_{\varrho_{1}} .
\end{aligned}
$$

Here,

$$
B_{\varrho}=\{\boldsymbol{x}:|\boldsymbol{x}|<\varrho\}, \quad S_{\varrho}=\{\boldsymbol{x}:|\boldsymbol{x}|=\varrho\},
$$

and

$$
A_{\varrho_{1}, \varrho_{2}}=\left\{\boldsymbol{x} \in \mathbb{R}^{2}: \varrho_{1}<|\boldsymbol{x}|<\varrho_{2}\right\} .
$$

The coefficients $k_{1}, k_{2}$ in $(2.1 \mathrm{e})$ are the thermal conductivity constants. $\Delta$ denotes the Laplacian and $f$ is a given function. The boundary of $A_{\varrho_{1}, \varrho_{2}}$ is $\partial A_{\varrho_{1}, \varrho_{2}}=S_{\varrho_{1}} \cup S_{\varrho_{2}}$. In (2.1e), $\partial / \partial n$ denotes the normal derivative in the outward direction to $B_{\varrho_{1}}$ on $S_{\varrho_{1}}$. 
2.2. MFS formulation. We treat the two subdomains separately: in $B_{\varrho_{1}}$ we approximate $u_{1}$ at a point $P$ by

$$
u_{1}^{N}(\boldsymbol{c}, \boldsymbol{Q} ; P)=\sum_{\nu=1}^{N} c_{\nu} \varphi\left(P, Q_{\nu}\right)
$$

and in $A_{\varrho_{1}, \varrho_{2}}$ we approximate $u_{2}$ at a point $P$ by

$$
u_{2}^{N}(\boldsymbol{d}, \boldsymbol{e}, \boldsymbol{S}, \boldsymbol{T} ; P)=\sum_{\nu=1}^{N} d_{\nu} \varphi\left(P, S_{\nu}\right)+\sum_{\nu=1}^{N} e_{\nu} \varphi\left(P, T_{\nu}\right)
$$

where the $2 N$-dimensional vectors $\boldsymbol{Q}, \boldsymbol{S}$, and $\boldsymbol{T}$ contain the coordinates of the singularities. In particular, the singularities described by $\boldsymbol{Q}$ lie outside $\bar{B}_{\varrho_{1}}$, whereas the singularities described by $\boldsymbol{S}$ and $\boldsymbol{T}$ lie outside the annulus $\bar{A}_{\varrho_{1}, \varrho_{2}}$.

The function $\varphi(P, Q)$ is a fundamental solution of the Laplacian $\Delta$, i.e.,

$$
-\Delta \varphi(\cdot, Q)=\delta_{Q},
$$

where $\delta_{Q}$ is the Dirac unit mass at $Q$. Equation (2.2) is satisfied in the sense of distributions. In particular, we take

$$
\varphi(P, Q)=-\frac{1}{2 \pi} \log |P-Q|,
$$

with $|P-Q|$ denoting the distance between the points $P$ and $Q$. The vectors $\boldsymbol{c}=$ $\left(c_{1}, \ldots, c_{N}\right), \boldsymbol{d}=\left(d_{1}, \ldots, d_{N}\right)$, and $\boldsymbol{e}=\left(e_{1}, \ldots, e_{N}\right)$ contain the coefficients of the fundamental solutions.

Specifically, the singularities $\boldsymbol{Q}=\left(Q_{1}, \ldots, Q_{N}\right)$ are placed on the circle of radius $R_{1}$, i.e., $S_{R_{1}}=\left\{\boldsymbol{x}:|\boldsymbol{x}|=R_{1}\right\}$, where $R_{1}>\varrho_{1}$, at the points

$$
Q_{j}=\left(R_{1} \cos \vartheta_{j}, R_{1} \sin \vartheta_{j}\right), \quad \vartheta_{j}=\frac{2 \pi(j-1+\alpha)}{N}, \quad j=1, \ldots, N,
$$

and the singularities $\boldsymbol{S}=\left(S_{1}, \ldots, S_{N}\right)$ and $\boldsymbol{T}=\left(T_{1}, \ldots, T_{N}\right)$ are placed on the circles $S_{R_{2}}=\left\{\boldsymbol{x}:|\boldsymbol{x}|=R_{2}\right\}$, and $S_{R_{3}}=\left\{\boldsymbol{x}:|\boldsymbol{x}|=R_{3}\right\}$, respectively, with $R_{2}<\varrho_{1}<\varrho_{2}<R_{3}$, at the points

$$
S_{j}=\left(R_{2} \cos \psi_{j}, R_{2} \sin \psi_{j}\right), \quad T_{j}=\left(R_{3} \cos \psi_{j}, R_{3} \sin \psi_{j}\right), \quad \psi_{j}=\frac{2 \pi(j-1+\beta)}{N},
$$

$j=1, \ldots, N$. The parameters $\alpha$ and $\beta$, where $\alpha, \beta \in\left[-\frac{1}{2}, \frac{1}{2}\right]$, describe rotations of the singularities. It has been observed that such rotations improve the approximation significantly, especially when the singularities are close to the boundary. (See [20, 21, $23,25])$.

Two sets of equally spaced collocation (or observation) points $\boldsymbol{P}^{1}=\left(P_{1}^{1}, \ldots, P_{N}^{1}\right)$ and $\boldsymbol{P}^{2}=\left(P_{1}^{2}, \ldots, P_{N}^{2}\right)$ are taken on the circles $S_{\varrho_{1}}$ and $S_{\varrho_{2}}$, respectively:

$$
P_{j}^{1}=\left(\varrho_{1} \cos \chi_{j}, \varrho_{1} \sin \chi_{j}\right), \quad P_{j}^{2}=\left(\varrho_{2} \cos \chi_{j}, \varrho_{2} \sin \chi_{j}\right), \quad \chi_{j}=\frac{2 \pi(j-1)}{N},
$$


where $j=1, \ldots, N$. The vectors of coefficients $\boldsymbol{c}, \boldsymbol{d}$, and $\boldsymbol{e}$ are determined from the collocation equations

$$
\begin{aligned}
u_{1}^{N}\left(\boldsymbol{c}, \boldsymbol{Q} ; P_{j}^{1}\right) & =u_{2}^{N}\left(\boldsymbol{d}, \boldsymbol{e}, \boldsymbol{S}, \boldsymbol{T} ; P_{j}^{1}\right), \\
k_{1} \frac{\partial}{\partial n} u_{1}^{N}\left(\boldsymbol{c}, \boldsymbol{Q} ; P_{j}^{1}\right) & =k_{2} \frac{\partial}{\partial n} u_{2}^{N}\left(\boldsymbol{d}, \boldsymbol{e}, \boldsymbol{S}, \boldsymbol{T} ; P_{j}^{1}\right), \\
u_{2}^{N}\left(\boldsymbol{d}, \boldsymbol{e}, \boldsymbol{S}, \boldsymbol{T} ; P_{j}^{2}\right) & =f\left(P_{j}^{2}\right),
\end{aligned}
$$

$j=1, \ldots, N$. Clearly, (2.3a), (2.3b), and (2.3c) correspond to (2.1d), (2.1e), and (2.1c), respectively. These yield the $3 N \times 3 N$ system

$$
H\left(\frac{c}{d}\right)=\left(\frac{0}{0}\right)
$$

where

$$
H=\left(\begin{array}{c|r|r|r|r}
\mathcal{A}\left(\boldsymbol{P}^{1}, \boldsymbol{Q}\right) & -\mathcal{A}\left(\boldsymbol{P}^{1}, \boldsymbol{S}\right) & -\mathcal{A}\left(\boldsymbol{P}^{1}, \boldsymbol{T}\right) \\
\hline k_{1} \mathcal{B}\left(\boldsymbol{P}^{1}, \boldsymbol{Q}\right) & -k_{2} \mathcal{B}\left(\boldsymbol{P}^{1}, \boldsymbol{S}\right) & -k_{2} \mathcal{B}\left(\boldsymbol{P}^{1}, \boldsymbol{T}\right) \\
\hline 0 & \mathcal{A}\left(\boldsymbol{P}^{2}, \boldsymbol{S}\right) & \mathcal{A}\left(\boldsymbol{P}^{2}, \boldsymbol{T}\right)
\end{array}\right)=\left(\begin{array}{c|c|c|}
H_{11} & H_{12} & H_{13} \\
\hline H_{21} & H_{22} & H_{23} \\
\hline H_{31} & H_{32} & H_{33}
\end{array}\right)
$$

and $\boldsymbol{f}=\left(f\left(P_{1}^{2}\right), \ldots, f\left(P_{N}^{2}\right)\right)$, with

$$
\begin{aligned}
(\mathcal{A}(\boldsymbol{V}, \boldsymbol{W}))_{\mu \nu} & =-\frac{1}{2 \pi} \log \left|V_{\mu}-W_{\nu}\right| \text { and } \\
(\mathcal{B}(\boldsymbol{V}, \boldsymbol{W}))_{\mu \nu} & =-\frac{\partial}{\partial n_{V_{\mu}}} \frac{1}{2 \pi} \log \left|V_{\mu}-W_{\nu}\right|
\end{aligned}
$$

for $\boldsymbol{V}=\left(V_{1}, \ldots, V_{N}\right), \boldsymbol{W}=\left(W_{1}, \ldots, W_{N}\right)$, and $\mu, \nu=1, \ldots, N$.

2.3. Matrix decomposition algorithm. All the blocks $H_{i j}, i, j=1,2,3$, of the coefficient matrix $H$ in system (2.4) are circulant. A square matrix $G$ is circulant (see [5]) if it has the form

$$
G=\left(\begin{array}{cccc}
g_{1} & g_{2} & \cdots & g_{N} \\
g_{N} & g_{1} & \cdots & g_{N-1} \\
\cdots & \cdots \cdots \cdots & \cdots \\
g_{2} & g_{3} & \cdots & g_{1}
\end{array}\right)
$$

and is denoted by $G=\operatorname{circ}\left(g_{1}, g_{2}, \ldots, g_{N}\right)$. Circulant matrices are diagonalizable by

$$
G=U^{*} D U,
$$

where

$$
D=\operatorname{diag}\left(\lambda_{1}, \ldots, \lambda_{N}\right)
$$

is a diagonal matrix whose diagonal elements are the eigenvalues of the matrix $G$, given by (see [5])

$$
\lambda_{j}=\sum_{\ell=1}^{N} \omega^{(j-1)(\ell-1)} g_{\ell}
$$


and

$$
U^{*}=\frac{1}{\sqrt{N}}\left(\begin{array}{ccccc}
1 & 1 & 1 & \ldots & 1 \\
1 & \omega & \omega^{2} & \ldots & \omega^{N-1} \\
1 & \omega^{2} & \omega^{4} & \ldots & \omega^{2 N-2} \\
\ldots & \ldots & \ldots & \ldots & \ldots \\
1 & \omega^{N-1} & \omega^{2(N-1)} & \ldots & \omega^{(N-1)(N-1)}
\end{array}\right)
$$

with $\omega=\mathrm{e}^{\frac{2 \pi \mathrm{i}}{N}}$. The matrix $U^{*}$, known as the Fourier matrix, is unitary.

It is obvious from (2.6) that the $\lambda_{j}$ can be obtained from the $g_{\ell}$ via discrete Fourier transforms. The blocks $H_{i j}, i, j=1,2,3$, of the system matrix $H$ can therefore be diagonalized by the Fourier matrix so that (2.4) may be written as

$$
\left(I_{3} \otimes U\right) H\left(I_{3} \otimes U^{*}\right)\left(I_{3} \otimes U\right)\left(\frac{\boldsymbol{c}}{\frac{\boldsymbol{d}}{\boldsymbol{e}}}\right)=\left(I_{3} \otimes U\right)\left(\frac{0}{\frac{0}{\boldsymbol{f}}}\right),
$$

where $I_{3}$ is the $3 \times 3$ identity matrix and $\otimes$ is the Kronecker tensor product. ${ }^{1}$

Thus system (2.8) can be written as

$$
\left(\begin{array}{c|c|c}
D_{11} & D_{12} & D_{13} \\
\hline D_{21} & D_{22} & D_{23} \\
\hline D_{31} & D_{32} & D_{33}
\end{array}\right)\left(\begin{array}{c}
\hat{\boldsymbol{c}} \\
\hline \hat{\boldsymbol{d}} \\
\hline \hat{\boldsymbol{e}}
\end{array}\right)=\left(\begin{array}{c}
0 \\
\hline 0 \\
\hline \hat{\boldsymbol{f}}
\end{array}\right),
$$

where

$$
\hat{c}=U c, \hat{d}=U d, \hat{e}=U e, \hat{f}=U \boldsymbol{f}
$$

and

$$
D_{i j}=U H_{i j} U^{*}=\operatorname{diag}\left(\lambda_{i j}^{1}, \ldots, \lambda_{i j}^{N}\right), \quad i, j=1,2,3 .
$$

The elements $\lambda_{i j}^{\ell}$ can be computed from (2.6), with the appropriate choice of $g_{\ell}$ 's.

Thus the linear system (2.9) reduces to $N$ independent $3 \times 3$ systems:

$$
\left(\begin{array}{ccc}
\lambda_{11}^{\ell} & \lambda_{12}^{\ell} & \lambda_{13}^{\ell} \\
\lambda_{21}^{\ell} & \lambda_{22}^{\ell} & \lambda_{23}^{\ell} \\
\lambda_{31}^{\ell} & \lambda_{32}^{\ell} & \lambda_{33}^{\ell}
\end{array}\right)\left(\begin{array}{c}
\hat{c}_{\ell} \\
\hat{d}_{\ell} \\
\hat{e}_{\ell}
\end{array}\right)=\left(\begin{array}{c}
0 \\
0 \\
\hat{f}_{\ell}
\end{array}\right), \quad \ell=1, \ldots, N .
$$

Finally, system (2.4) can be solved efficiently using the following algorithm:

\footnotetext{
${ }^{1}$ The tensor (or Kronecker) product of the $m \times n$ matrix $V$ and the $\ell \times k$ matrix $W$ is the $m \ell \times n k$ matrix

$$
V \otimes W=\left(\begin{array}{cccc}
v_{11} W & v_{12} W & \cdots & v_{1 n} W \\
v_{21} W & v_{22} W & \cdots & v_{2 n} W \\
\ldots \ldots \ldots & \ldots \ldots \ldots \ldots \ldots & \ldots & \ldots \\
v_{m 1} W & v_{m 2} W & \cdots & v_{m n} W
\end{array}\right)
$$

Basic properties are (i) $V \otimes(W \otimes Z)=(V \otimes W) \otimes Z$, (ii) $\left(V_{1} \otimes W_{1}\right)\left(V_{2} \otimes W_{2}\right)=\left(V_{1} V_{2}\right) \otimes\left(W_{1} W_{2}\right)$, and (iii) $V \otimes(W+Z)=V \otimes W+V \otimes Z$. A comprehensive list of properties of the tensor product can be found in [5, pp. 22-23] or [17, pp. 597-598].
} 
Step 1: Compute $\hat{\boldsymbol{f}}=U \boldsymbol{f}$.

Step 2: Evaluate the (diagonal) elements the diagonal matrices $D_{\mu \nu}, \mu, \nu=1,2,3$, using formula (2.6).

Step 3: Solve the $N$ systems of order 3 in (2.10) to determine the vectors $\hat{\boldsymbol{c}}, \hat{\boldsymbol{d}}$, and $\hat{e}$.

Step 4: Compute the vectors $\boldsymbol{c}=U^{*} \hat{\boldsymbol{c}}, \boldsymbol{d}=U^{*} \hat{\boldsymbol{d}}$, and $\boldsymbol{e}=U^{*} \hat{\boldsymbol{e}}$.

Cost. (i) In Steps 1 and 4 , because of the form of the matrices $U$ and $U^{*}$, the operations can be carried out via FFTs at a cost of $\mathcal{O}(N \log N)$ operations.

(ii) FFTs can also be used for the evaluation of the diagonal matrices in Step 2 at a cost of $\mathcal{O}(N \log N)$.

(iii) In Step 3, we need to solve $N$ complex linear systems of order 3 which can be done at a cost of $\mathcal{O}(N)$ operations.

\section{The three-dimensional case.}

3.1. The problem. We consider the bimaterial problem in $\mathbb{R}^{3}$,

$$
\begin{array}{lll}
\Delta u_{1}=0 & \text { in } & \Omega_{1}, \\
\Delta u_{2}=0 & \text { in } & \Omega_{2} \backslash \bar{\Omega}_{1},
\end{array}
$$

where $\bar{\Omega}_{1} \subset \Omega_{2}$, subject to the boundary condition

$$
u_{2}=f \quad \text { on } \quad \partial \Omega_{2}
$$

and the interface conditions

$$
\begin{aligned}
u_{1} & =u_{2} \quad \text { and } \\
k_{1} \frac{\partial u_{1}}{\partial n} & =k_{2} \frac{\partial u_{2}}{\partial n} \quad \text { on } \quad \partial \Omega_{1} .
\end{aligned}
$$

The regions $\Omega_{1}, \Omega_{2} \subset \mathbb{R}^{3}$ are axisymmetric, which means that they are formed by rotating regions $\Omega_{1}^{\prime}, \Omega_{2}^{\prime} \subset \mathbb{R}^{2}\left(\Omega_{1}^{\prime} \subset \Omega_{2}^{\prime}\right)$, respectively, about the $z$-axis.

3.2. MFS formulation. As in the two-dimensional case, we treat the two subdomains separately: in $\Omega_{1}$ we approximate $u_{1}$ at a point $P$ by

$$
u_{1}^{M, N}(\boldsymbol{c}, \boldsymbol{Q} ; P)=\sum_{\mu=1}^{M} \sum_{\nu=1}^{N} c_{\mu, \nu} \Phi\left(P, Q_{\mu, \nu}\right)
$$

and in $\Omega_{2} \backslash \bar{\Omega}_{1}, u_{2}$ at a point $P$ is approximated by

$$
u_{2}^{M, N}(\boldsymbol{d}, \boldsymbol{e}, \boldsymbol{S}, \boldsymbol{T} ; P)=\sum_{\mu=1}^{M} \sum_{\nu=1}^{N} d_{\mu, \nu} \Phi\left(P, S_{\mu, \nu}\right)+\sum_{\mu=1}^{M} \sum_{\nu=1}^{N} e_{\mu, \nu} \Phi\left(P, T_{\mu, \nu}\right) .
$$

Here

$$
\begin{aligned}
\boldsymbol{c} & =\left(c_{11}, c_{12}, \ldots, c_{1 N}, \ldots, c_{M 1}, \ldots, c_{M N}\right)^{T}, \\
\boldsymbol{d} & =\left(d_{11}, d_{12}, \ldots, d_{1 N}, \ldots, d_{M 1}, \ldots, d_{M N}\right)^{T}, \\
\boldsymbol{e} & =\left(e_{11}, e_{12}, \ldots, e_{1 N}, \ldots, e_{M 1}, \ldots, e_{M N}\right)^{T},
\end{aligned}
$$


and $\boldsymbol{Q}, \boldsymbol{S}, \boldsymbol{T}$ are $3 M N$-vectors containing the coordinates of the singularities $Q_{\mu, \nu}$, $S_{\mu, \nu}, T_{\mu, \nu}, \mu=1, \ldots, M, \nu=1, \ldots, N$. The singularities described by $\boldsymbol{Q}$ lie outside $\bar{\Omega}_{1}$, whereas the singularities described by $\boldsymbol{S}$ and $\boldsymbol{T}$ lie outside $\bar{\Omega}_{2} \backslash \Omega_{1}$. More specifically, the singularities $\boldsymbol{S}$ lie in $\Omega_{1}$ and the singularities $\boldsymbol{T}$ lie outside $\bar{\Omega}_{2}$.

The function $\Phi(P, Q)$ is a fundamental solution of Laplace's equation in $\mathbb{R}^{3}$ given by

$$
\Phi(P, Q)=\frac{1}{4 \pi|P-Q|}
$$

with $|P-Q|$, as before, denoting the distance between the points $P$ and $Q$.

Two sets of $M N$ collocation points $\left\{P_{m, n}^{1}\right\}_{m=1, n=1}^{M, N}$ and $\left\{P_{m, n}^{2}\right\}_{m=1, n=1}^{M, N}$ are chosen on $\partial \Omega_{1}$ and $\partial \Omega_{2}$, respectively, in the following way.

We first choose $N$ points on each of the boundaries $\partial \Omega_{1}^{\prime}$ and $\partial \Omega_{2}^{\prime}$. These are described by their cartesian coordinates $\left(r_{n}^{P^{1}}, z_{n}^{P^{1}}\right), n=1, \ldots, N$, and $\left(r_{n}^{P^{2}}, z_{n}^{P^{2}}\right)$, $n=1, \ldots, N$, in $\Omega_{1}^{\prime}$ and $\Omega_{2}^{\prime}$, respectively. Here $r_{n}^{P^{i}}$ denotes the horizontal distance from the axis of rotation, and $z_{n}^{P^{i}}$ denotes the $z$-coordinate. The points on $\partial \Omega_{i}$, $i=1,2$, are taken to be

$$
x_{P_{m, n}^{i}}=r_{n}^{P^{i}} \cos \varphi_{m}, y_{P_{m, n}^{i}}=r_{n}^{P^{i}} \sin \varphi_{m}, \quad z_{P_{m, n}^{i}}=z_{n}^{P^{i}},
$$

where $\varphi_{m}=\frac{2(m-1) \pi}{M}, m=1, \ldots, M$.

Similarly, we choose three sets of $M N$ singularities $\left\{Q_{\mu, \nu}\right\}_{\mu=1, \nu=1}^{M, N},\left\{S_{\mu, \nu}\right\}_{\mu=1, \nu=1}^{M, N}$, and $\left\{T_{\mu, \nu}\right\}_{\mu=1, \nu=1}^{M, N}$ given by

$$
\begin{aligned}
& x_{Q_{\mu, \nu}}=r_{\nu}^{Q} \cos \psi_{\mu}, \quad y_{Q_{\mu, \nu}}=r_{\nu}^{Q} \sin \psi_{\mu}, \quad z_{Q_{\mu, \nu}}=z_{\nu}^{Q}, \\
& x_{S_{\mu, \nu}}=r_{\nu}^{S} \cos \chi_{\mu}, \quad y_{S_{\mu, \nu}}=r_{\nu}^{S} \sin \chi_{\mu}, \quad z_{S_{\mu, \nu}}=z_{\nu}^{S} \\
& x_{T_{\mu, \nu}}=r_{\nu}^{T} \cos \chi_{\mu}, \quad y_{T_{\mu, \nu}}=r_{\nu}^{T} \sin \chi_{\mu}, \quad z_{T_{\mu, \nu}}=z_{\nu}^{T},
\end{aligned}
$$

where

$$
\psi_{\mu}=\frac{2(\mu-1+\alpha) \pi}{M}, \quad \chi_{\mu}=\frac{2(\mu-1+\beta) \pi}{M}, \quad \mu=1, \ldots, M .
$$

The parameters $\alpha, \beta \in\left[-\frac{1}{2}, \frac{1}{2}\right]$ prescribe the rotations of the singularities in the azimuthal direction.

The coefficients $\boldsymbol{c}, \boldsymbol{d}$, and $\boldsymbol{e}$ are determined so that the interface and boundary conditions are satisfied at the boundary points $\left\{P_{m, n}^{1}\right\}_{m=1, n=1}^{M, N}$ and $\left\{P_{m, n}^{2}\right\}_{m=1, n=1}^{M, N}$, respectively,

$$
\begin{array}{r}
u_{1}^{M, N}\left(\boldsymbol{c}, \boldsymbol{Q} ; P_{m n}^{1}\right)-u_{2}^{M, N}\left(\boldsymbol{d}, \boldsymbol{e}, \boldsymbol{S}, \boldsymbol{T} ; P_{m n}^{1}\right)=0 \\
k_{1} \frac{\partial}{\partial n} u_{1}^{M, N}\left(\boldsymbol{c}, \boldsymbol{Q} ; P_{m n}^{1}\right)-k_{2} \frac{\partial}{\partial n} u_{2}^{M, N}\left(\boldsymbol{d}, \boldsymbol{e}, \boldsymbol{S}, \boldsymbol{T} ; P_{m n}^{1}\right)=0 \\
u_{2}^{M, N}\left(\boldsymbol{d}, \boldsymbol{e}, \boldsymbol{S}, \boldsymbol{T} ; P_{m, n}^{2}\right)=f\left(P_{m, n}^{2}\right),
\end{array}
$$

$m=1, \ldots, M, n=1, \ldots, N$. These equations yield a $3 M N \times 3 M N$ linear system of the form

$$
H\left(\frac{c}{d}\right)=\left(\frac{0}{e}\right),
$$


where

$$
H=\left(\begin{array}{c|r|r|r|r}
\mathcal{A}\left(\boldsymbol{P}^{1}, \boldsymbol{Q}\right) & -\mathcal{A}\left(\boldsymbol{P}^{1}, \boldsymbol{S}\right) & -\mathcal{A}\left(\boldsymbol{P}^{1}, \boldsymbol{T}\right) \\
\hline k_{1} \mathcal{B}\left(\boldsymbol{P}^{1}, \boldsymbol{Q}\right) & -k_{2} \mathcal{B}\left(\boldsymbol{P}^{1}, \boldsymbol{S}\right) & -k_{2} \mathcal{B}\left(\boldsymbol{P}^{1}, \boldsymbol{T}\right) \\
\hline 0 & \mathcal{A}\left(\boldsymbol{P}^{2}, \boldsymbol{S}\right) & \mathcal{A}\left(\boldsymbol{P}^{2}, \boldsymbol{T}\right)
\end{array}\right)=\left(\begin{array}{c|c|c}
H_{11} & H_{12} & H_{13} \\
\hline H_{21} & H_{22} & H_{23} \\
\hline H_{31} & H_{32} & H_{33}
\end{array}\right) .
$$

Here $\boldsymbol{f}=\left(f_{m n}\right)_{m=1, n=1}^{M, N}$ and the $M N \times M N$ matrices are given by

$$
\begin{aligned}
(\mathcal{A}(\boldsymbol{V}, \boldsymbol{W}))_{(m-1) N+n,(\mu-1) N+\nu} & =\frac{1}{4 \pi\left|V_{m, n}-W_{\mu, \nu}\right|}, \\
(\mathcal{B}(\boldsymbol{V}, \boldsymbol{W}))_{(m-1) N+n,(\mu-1) N+\nu} & =\frac{\partial}{\partial n_{V_{m, n}}}\left\{\frac{1}{4 \pi\left|V_{m, n}-W_{\mu, \nu}\right|}\right\}
\end{aligned}
$$

for

$$
\begin{aligned}
\boldsymbol{V} & =\left(V_{11}, V_{12}, \ldots, V_{1 N}, \ldots, V_{M 1}, \ldots, V_{M N}\right)^{T} \\
\boldsymbol{W} & =\left(W_{11}, W_{12}, \ldots, W_{1 N}, \ldots, W_{M 1}, \ldots, W_{M N}\right)^{T}
\end{aligned}
$$

and $m, \mu=1, \ldots, M$ and $n, \nu=1, \ldots, N$.

3.3. Matrix decomposition algorithm. Each of the nine blocks $H_{i j}, i, j=$ $1,2,3$, of the coefficient matrix $H$ in (3.4) has a block circulant structure $[8,22]$ of the form

$$
H_{i j}=\left(\begin{array}{c|c|c|c}
H_{i j}^{1} & H_{i j}^{2} & \cdots & H_{i j}^{M} \\
\hline H_{i j}^{M} & H_{i j}^{1} & \cdots & H_{i j}^{M-1} \\
\hline \vdots & \vdots & \ddots & \vdots \\
\hline H_{i j}^{2} & H_{i j}^{3} & \cdots & H_{i j}^{1}
\end{array}\right),
$$

where the matrices $H_{i j}^{\ell}, \ell=1, \ldots, M, i, j=1,2,3$, are $N \times N$ matrices defined by

$$
\begin{array}{r}
\left(H_{11}^{\ell}\right)_{n, \nu}=\frac{1}{4 \pi\left|P_{1, n}^{1}-Q_{\ell, \nu}\right|}, \quad\left(H_{12}^{\ell}\right)_{n, \nu}=-\frac{1}{4 \pi\left|P_{1, n}^{1}-S_{\ell, \nu}\right|}, \\
\left(H_{13}^{\ell}\right)_{n, \nu}=-\frac{1}{4 \pi\left|P_{1, n}^{1}-T_{\ell, \nu}\right|}, \quad\left(H_{21}^{\ell}\right)_{n, \nu}=k_{1} \frac{\partial}{\partial n_{P_{1, n}^{1}}}\left\{\frac{1}{4 \pi\left|P_{1, n}^{1}-Q_{\ell, \nu}\right|}\right\}, \\
\left(H_{22}^{\ell}\right)_{n, \nu}=-k_{2} \frac{\partial}{\partial n_{P_{1, n}^{1}}}\left\{\frac{1}{4 \pi\left|P_{1, n}^{1}-S_{\ell, \nu}\right|}\right\}, \quad\left(H_{23}^{\ell}\right)_{n, \nu}=-k_{2} \frac{\partial}{\partial n_{P_{1, n}^{1}}}\left\{\frac{1}{4 \pi\left|P_{1, n}^{1}-T_{\ell, \nu}\right|}\right\}, \\
\left(H_{31}^{\ell}\right)_{n, \nu}=0, \quad\left(H_{32}^{\ell}\right)_{n, \nu}=\frac{1}{4 \pi\left|P_{1, n}^{2}-S_{\ell, \nu}\right|}, \quad\left(H_{33}^{\ell}\right)_{n, \nu}=\frac{1}{4 \pi\left|P_{1, n}^{2}-T_{\ell, \nu}\right|},
\end{array}
$$

$n, \nu=1, \ldots, N$. Because of the block circulant structure of the matrices $H_{i j}$, following a technique similar to the ones employed in $[8,22]$, for every $i, j=1,2,3$, we have that

$$
H_{i j}=\sum_{\ell=1}^{N} \mathcal{P}^{\ell-1} \otimes H_{i j}^{\ell}
$$


where the matrix $\mathcal{P}$ is the $M \times M$ permutation matrix $\mathcal{P}=\operatorname{circ}(0,1,0, \ldots, 0)$ and $\mathcal{P}^{0}=I_{M}$. Thus

$$
\begin{aligned}
H & =\left(\begin{array}{l|l|l}
H_{11} & H_{12} & H_{13} \\
\hline H_{21} & H_{22} & H_{23} \\
\hline H_{31} & H_{32} & H_{33}
\end{array}\right) \\
& =\left(\begin{array}{c|c|c|c}
\sum_{\ell=1}^{M} \mathcal{P}^{\ell-1} \otimes H_{11}^{\ell} & \sum_{\ell=1}^{M} \mathcal{P}^{\ell-1} \otimes H_{12}^{\ell} & \sum_{\ell=1}^{M} \mathcal{P}^{\ell-1} \otimes H_{13}^{\ell} \\
\hline \sum_{\ell=1}^{M} \mathcal{P}^{\ell-1} \otimes H_{21}^{\ell} & \sum_{\ell=1}^{M} \mathcal{P}^{\ell-1} \otimes H_{22}^{\ell} & \sum_{\ell=1}^{M} \mathcal{P}^{\ell-1} \otimes H_{23}^{\ell} \\
\hline \sum_{\ell=1}^{M} \mathcal{P}^{\ell-1} \otimes H_{31}^{\ell} & \sum_{\ell=1}^{M} \mathcal{P}^{\ell-1} \otimes H_{32}^{\ell} & \sum_{\ell=1}^{M} \mathcal{P}^{\ell-1} \otimes H_{33}^{\ell}
\end{array}\right) \\
& =\sum_{\ell=1}^{M}\left(\begin{array}{|l|l|l}
\mathcal{P}^{\ell-1} \otimes H_{11}^{\ell} & \mathcal{P}^{\ell-1} \otimes H_{12}^{\ell} & \mathcal{P}^{\ell-1} \otimes H_{13}^{\ell} \\
\hline \mathcal{P}^{\ell-1} \otimes H_{21}^{\ell} & \mathcal{P}^{\ell-1} \otimes H_{22}^{\ell} & \mathcal{P}^{\ell-1} \otimes H_{23}^{\ell} \\
\hline \mathcal{P}^{\ell-1} \otimes H_{31}^{\ell} & \mathcal{P}^{\ell-1} \otimes H_{32}^{\ell} & \mathcal{P}^{\ell-1} \otimes H_{33}^{\ell}
\end{array}\right) .
\end{aligned}
$$

From [5], the matrices $\mathcal{P}^{\ell}$ can be diagonalized as

$$
\mathcal{P}^{\ell}=U^{*} E^{\ell} U,
$$

where $U$ is defined from $(2.7)$ and $E=\operatorname{diag}\left(1, \omega, \omega^{2}, \ldots, \omega^{M-1}\right)$, with $\omega=\mathrm{e}^{2 \pi \mathrm{i} / M}$. Premultiplying system (3.4) by

$$
\left(\begin{array}{c|c|c}
U & 0 & 0 \\
\hline 0 & U & 0 \\
\hline 0 & 0 & U
\end{array}\right) \otimes I_{N}=I_{3} \otimes U \otimes I_{N}
$$

yields

$$
\left(I_{3} \otimes U \otimes I_{N}\right) H\left(I_{3} \otimes U^{*} \otimes I_{N}\right)\left(I_{3} \otimes U \otimes I_{N}\right)\left(\frac{\frac{\boldsymbol{c}}{\boldsymbol{d}}}{\boldsymbol{e}}\right)=\left(I_{3} \otimes U \otimes I_{N}\right)\left(\frac{0}{\frac{0}{\boldsymbol{f}}}\right)
$$

(using the fact that $\left.\left(I_{3} \otimes U^{*} \otimes I_{N}\right)\left(I_{3} \otimes U \otimes I_{N}\right)=I_{3 M N}\right)$ or

$$
\tilde{H}\left(\begin{array}{l}
\frac{\tilde{\boldsymbol{c}}}{\tilde{\boldsymbol{d}}} \\
\hline \tilde{\boldsymbol{e}}
\end{array}\right)=\left(\begin{array}{l}
\frac{0}{0} \\
\hline \tilde{f}
\end{array}\right)
$$

where

$$
\tilde{H}=\left(I_{3} \otimes U \otimes I_{N}\right) H\left(I_{3} \otimes U^{*} \otimes I_{N}\right)
$$

and

$$
\tilde{\boldsymbol{c}}=\left(U \otimes I_{N}\right) \boldsymbol{c}, \quad \tilde{\boldsymbol{d}}=\left(U \otimes I_{N}\right) \boldsymbol{d}, \quad \tilde{\boldsymbol{e}}=\left(U \otimes I_{N}\right) \boldsymbol{e}, \quad \tilde{\boldsymbol{f}}=\left(U \otimes I_{N}\right) \boldsymbol{f} .
$$

From (3.5)

$$
\tilde{H}=\sum_{\ell=1}^{M}\left(I_{3} \otimes U \otimes I_{N}\right)\left(\begin{array}{c|c|c}
\mathcal{P}^{\ell-1} \otimes H_{11}^{\ell} & \mathcal{P}^{\ell-1} \otimes H_{12}^{\ell} & \mathcal{P}^{\ell-1} \otimes H_{13}^{\ell} \\
\hline \mathcal{P}^{\ell-1} \otimes H_{21}^{\ell} & \mathcal{P}^{\ell-1} \otimes H_{22}^{\ell} & \mathcal{P}^{\ell-1} \otimes H_{23}^{\ell} \\
\hline \mathcal{P}^{\ell-1} \otimes H_{31}^{\ell} & \mathcal{P}^{\ell-1} \otimes H_{32}^{\ell} & \mathcal{P}^{\ell-1} \otimes H_{33}^{\ell}
\end{array}\right)\left(I_{3} \otimes U^{*} \otimes I_{N}\right),
$$


and using the fact that

$$
\left(U \otimes I_{N}\right)\left(\mathcal{P}^{\ell-1} \otimes H_{i j}^{\ell}\right)\left(U^{*} \otimes I_{N}\right)=E^{\ell-1} \otimes H_{i j}^{\ell},
$$

we obtain

$$
\tilde{H}=\sum_{\ell=1}^{M}\left(\begin{array}{c|c|c}
E^{\ell-1} \otimes H_{11}^{\ell} & E^{\ell-1} \otimes H_{12}^{\ell} & E^{\ell-1} \otimes H_{13}^{\ell} \\
\hline E^{\ell-1} \otimes H_{21}^{\ell} & E^{\ell-1} \otimes H_{22}^{\ell} & E^{\ell-1} \otimes H_{23}^{\ell} \\
\hline E^{\ell-1} \otimes H_{31}^{\ell} & E^{\ell-1} \otimes H_{32}^{\ell} & E^{\ell-1} \otimes H_{33}^{\ell}
\end{array}\right) .
$$

Thus, system (3.6) can be written as

$$
\sum_{\ell=1}^{M}\left(\begin{array}{c|c|c}
E^{\ell-1} \otimes H_{11}^{\ell} & E^{\ell-1} \otimes H_{12}^{\ell} & E^{\ell-1} \otimes H_{13}^{\ell} \\
\hline E^{\ell-1} \otimes H_{21}^{\ell} & E^{\ell-1} \otimes H_{22}^{\ell} & E^{\ell-1} \otimes H_{23}^{\ell} \\
\hline E^{\ell-1} \otimes H_{31}^{\ell} & E^{\ell-1} \otimes H_{32}^{\ell} & E^{\ell-1} \otimes H_{33}^{\ell}
\end{array}\right)\left(\begin{array}{c}
\tilde{\boldsymbol{c}} \\
\hline \tilde{\boldsymbol{d}} \\
\hline \tilde{\boldsymbol{e}}
\end{array}\right)=\left(\begin{array}{c}
0 \\
\hline 0 \\
\hline \tilde{\boldsymbol{f}}
\end{array}\right) .
$$

Since the matrix $E$ is diagonal, each block $E^{\ell-1} \otimes H_{i j}^{\ell}$ is block diagonal, i.e.,

$$
E^{\ell-1} \otimes H_{i j}^{\ell}=\operatorname{diag}\left(H_{i j}^{\ell}, \omega^{\ell-1} H_{i j}^{\ell}, \ldots, \omega^{(\ell-1)(M-1)} H_{i j}^{\ell}\right),
$$

as are the blocks of the matrix $\tilde{H}$ :

$$
\begin{aligned}
\sum_{\ell=1}^{M} E^{\ell-1} \otimes H_{i j}^{\ell} & =\operatorname{diag}\left(\sum_{\ell=1}^{M} H_{i j}^{\ell}, \sum_{\ell=1}^{M} \omega^{\ell-1} H_{i j}^{\ell}, \ldots, \sum_{\ell=1}^{M} \omega^{(\ell-1)(M-1)} H_{i j}^{\ell}\right) \\
& =\operatorname{diag}\left(\tilde{H}_{i j}^{1}, \tilde{H}_{i j}^{2}, \ldots, \tilde{H}_{i j}^{M}\right)
\end{aligned}
$$

where

$$
\tilde{H}_{i j}^{m}=\sum_{\ell=1}^{M} \omega^{(\ell-1)(m-1)} H_{i j}^{\ell}, \quad m=1, \ldots, M, \quad i, j=1,2,3 .
$$

System (3.4) thus reduces to the $M$ independent $3 N \times 3 N$ systems:

$$
\left(\begin{array}{c|c|c}
\tilde{H}_{11}^{m} & \tilde{H}_{12}^{m} & \tilde{H}_{13}^{m} \\
\hline \tilde{H}_{21}^{m} & \tilde{H}_{22}^{m} & \tilde{H}_{23}^{m} \\
\hline \tilde{H}_{31}^{m} & \tilde{H}_{32}^{m} & \tilde{H}_{33}^{m}
\end{array}\right)\left(\begin{array}{c}
\tilde{\boldsymbol{c}}_{m} \\
\hline \tilde{\boldsymbol{d}}_{m} \\
\hline \tilde{\boldsymbol{e}}_{m}
\end{array}\right)=\left(\begin{array}{c}
0 \\
\hline 0 \\
\hline \tilde{\boldsymbol{f}}_{m}
\end{array}\right), \quad m=1, \ldots, M,
$$

where

$$
\begin{aligned}
& \tilde{\boldsymbol{c}}_{m}=\left(\tilde{c}_{m 1}, \tilde{c}_{m 2}, \cdots, \tilde{c}_{m N}\right)^{T}, \quad \tilde{\boldsymbol{d}}_{m}=\left(\tilde{d}_{m 1}, \tilde{d}_{m 2}, \cdots, \tilde{d}_{m N}\right)^{T}, \\
& \tilde{\boldsymbol{e}}_{m}=\left(\tilde{e}_{m 1}, \tilde{e}_{m 2}, \cdots, \tilde{e}_{m N}\right)^{T}, \quad \tilde{\boldsymbol{f}}_{m}=\left(\tilde{f}_{m 1}, \tilde{f}_{m 2}, \cdots, \tilde{f}_{m N}\right)^{T} .
\end{aligned}
$$

Once $\tilde{\boldsymbol{c}}, \tilde{\boldsymbol{d}}$, and $\tilde{\boldsymbol{e}}$ are computed, the vectors $\boldsymbol{c}, \boldsymbol{d}$, and $\boldsymbol{e}$ can be calculated from

$$
\boldsymbol{c}=\left(U^{*} \otimes I_{N}\right) \tilde{\boldsymbol{c}}, \quad \boldsymbol{d}=\left(U^{*} \otimes I_{N}\right) \tilde{\boldsymbol{d}}, \quad \boldsymbol{e}=\left(U^{*} \otimes I_{N}\right) \tilde{\boldsymbol{e}} .
$$

System (3.8) can thus be solved efficiently using the following algorithm:

Step 1: Compute $\tilde{\boldsymbol{f}}=\left(U \otimes I_{N}\right) \boldsymbol{f}$.

Step 2: Construct the matrices $\tilde{H}_{i j}^{m}, i, j=1,2,3, m=1, \ldots, M$, from formula (3.7). 
Step 3: Solve the $M$ systems of order $3 N$ in (3.8).

Step 4: Compute $\boldsymbol{c}, \boldsymbol{d}$, and $\boldsymbol{e}$ from (3.9).

Cost.

(i) In Steps 1 and 4, because of the form of the matrices $U$ and $U^{*}$, the operations can be carried out via FFTs and inverse FFTs at a cost of $\mathcal{O}(N M \log M)$.

(ii) In Step 2, for each $r, s=1, \ldots, N$, we need to perform an $M$-dimensional inverse FFT, in order to compute the entries $\left(\tilde{H}_{i j}^{1}\right)_{r s}, \ldots,\left(\tilde{H}_{i j}^{M}\right)_{r s}$. This can be done at a cost of $\mathcal{O}\left(N^{2} M \log M\right)$.

(iii) In Step 3, we need to solve $M$ complex linear systems of order $3 N$. This can be done using an LU-factorization with partial pivoting at a cost of $\mathcal{O}\left(M N^{3}\right)$ operations.

\section{Numerical experiments.}

4.1. Two-dimensional case. In this subsection, we test numerically the algorithms proposed in section 2 in the case of problem (2.1). Since both the exact solutions $u_{1}, u_{2}$ and approximate solutions $u_{1}^{N}, u_{2}^{N}$ are harmonic in $\mathfrak{O}_{1}=B_{\varrho_{1}}$ and $\mathfrak{O}_{2}=A_{\varrho_{1}, \varrho_{2}}$, respectively, the maximum principle applies to their difference, and thus

$$
\sup _{\boldsymbol{x} \in \mathfrak{O}_{j}}\left|u_{j}^{N}(\boldsymbol{x})-u_{j}(\boldsymbol{x})\right|=\sup _{\boldsymbol{x} \in \partial \mathfrak{O}_{j}}\left|u_{j}^{N}(\boldsymbol{x})-u_{j}(\boldsymbol{x})\right|, \quad j=1,2 .
$$

In all of our experiments, we calculated the maximum relative error of the problem

$$
E=\max \left\{E_{1}, E_{2}\right\}
$$

where

$$
E_{j}=\frac{\sup _{\boldsymbol{x} \in \partial \mathfrak{O}_{j}}\left|u_{j}^{N}(\boldsymbol{x})-u_{j}(\boldsymbol{x})\right|}{\sup _{\boldsymbol{x} \in \partial \mathfrak{O}_{j}}\left|u_{j}(\boldsymbol{x})\right|}, \quad j=1,2 .
$$

The maximum relative error was calculated on uniform grids on the boundaries of the two regions, namely, at the points $\left\{V_{\ell}^{j}\right\}_{\ell=1, \ldots, L}^{j=1,2}$ given by

$$
V_{\ell}^{j}=\left(\varrho_{j} \cos \vartheta_{\ell}, \varrho_{j} \sin \vartheta_{\ell}\right), \quad \vartheta_{\ell}=\frac{2 \pi}{L}(\ell-1) .
$$

In most experiments, the parameter $L$ was taken to be equal to 1001 .

In our numerical experiments we took the boundary data in (2.1c) to represent Fourier frequencies, namely, $f=\varrho_{2}^{m} \mathrm{e}^{\mathrm{i} m \vartheta}$, where $m$ is a nonnegative integer. The exact solution of this problem, for $m>0$, is of the form

$$
u_{1}(r, \vartheta)=A r^{m} \mathrm{e}^{\mathrm{i} m \vartheta}, \quad u_{2}(r, \vartheta)=B r^{m} \mathrm{e}^{\mathrm{i} m \vartheta}+\Gamma r^{-m} \mathrm{e}^{\mathrm{i} m \vartheta},
$$

where $A, B$, and $\Gamma$ can be uniquely determined from (2.1c)-(2.1e),

$$
\begin{aligned}
& A=\frac{2 k_{2} \varrho_{2}^{2 m}}{k_{1}\left(\varrho_{2}^{2 m}-\varrho_{1}^{2 m}\right)+k_{2}\left(\varrho_{1}^{2 m}+\varrho_{2}^{2 m}\right)}, \\
& B=\frac{\varrho_{2}^{2 m}\left(k_{1}+k_{2}\right)}{k_{1}\left(\varrho_{2}^{2 m}-\varrho_{1}^{2 m}\right)+k_{2}\left(\varrho_{1}^{2 m}+\varrho_{2}^{2 m}\right)}, \\
& \Gamma=\frac{k_{2}-k_{1}}{k_{1}\left(\varrho_{2}^{2 m}-\varrho_{1}^{2 m}\right)+k_{2}\left(\varrho_{1}^{2 m}+\varrho_{2}^{2 m}\right)} .
\end{aligned}
$$

When $m=0$, the solution is $u_{1}=u_{2}=1$. We also chose $\varrho_{1}=1, \varrho_{2}=3, k_{2} / k_{1}=2$. 


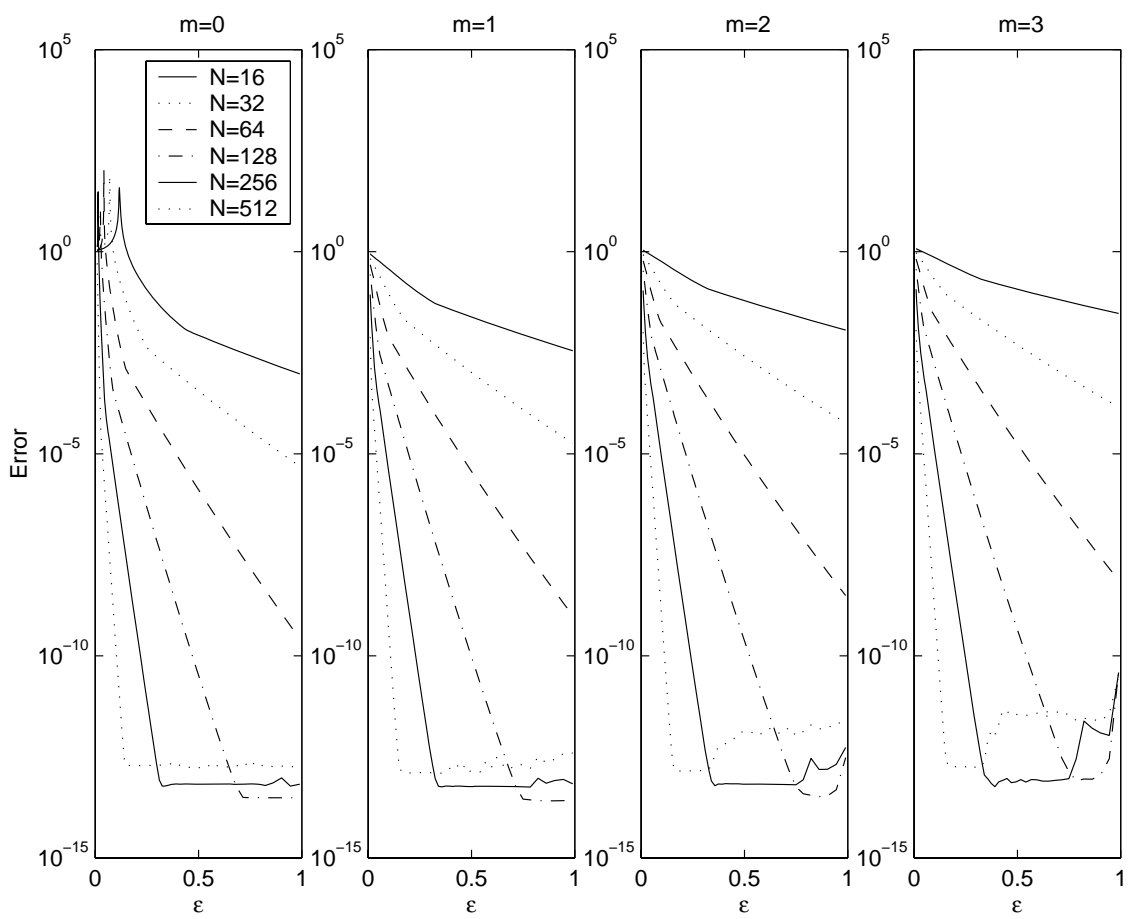

FIG. 1. Semilog-plot of the error versus $\varepsilon$ in Example 1 for different values of $N$ when $f=$ $\varrho_{2}^{m} \mathrm{e}^{\mathrm{i} m \vartheta}, m=0,1,2,3$.

The FFT operations were performed using the $\mathrm{NAG}^{2}$ routines C06FPF, C06FQF, and C06FRF. All numerical experiments were carried out on an IBM RS6000 (375 $\mathrm{MHz}$ ) workstation.

Figure 1. We present the logarithm of $E$, as defined by (4.1a) and (4.1b), versus the distance $\varepsilon$ of the three circles $S_{R_{j}}, j=1,2,3$ describing the pseudoboundaries from the corresponding boundaries, $S_{\varrho_{1}}$ and $S_{\varrho_{2}}$. That is,

$$
R_{1}=\varrho_{1}+\varepsilon, \quad R_{2}=\varrho_{1}-\varepsilon, \quad R_{3}=\varrho_{2}+\varepsilon .
$$

Here we consider the unrotated case, i.e., $\alpha=\beta=0$. We consider the cases $m=$ $0,1,2,3$ with $N=16,32,64,128,256$ and 512 .

We observe that, for a fixed $m$ and a fixed $N$, as $\varepsilon \in(0,1)$ grows, the accuracy improves exponentially fast. Further, for fixed $\varepsilon$ and fixed $m$, the error decays exponentially as $N$ grows. Also, for fixed $N$ and $\varepsilon$, as $m$ increases, the error increases.

The cusps in the case $m=0$ occur because the coefficient matrix in (2.10), when $\ell=1$, becomes singular for the values of $\varepsilon$ where the cusps occur. When $\ell \neq 1$, no such singularity was observed. Since only the solution of system (2.10) for $\ell=m+1$ contributes to the MFS solution corresponding to $m$, this phenomenon is not observed for $m \neq 0$. The analyses of similar observations, in the case of the Dirichlet problem in the disk and the annulus, are reported in [23, 25], respectively.

Note that as $N$ and $\varepsilon$ increase, the errors become close to machine accuracy.

\footnotetext{
${ }^{2}$ Numerical Analysis Group (NAG) Library Mark 20, NAG Ltd, Wilkinson House, Jordan Hill Road, Oxford, UK, 2001.
} 

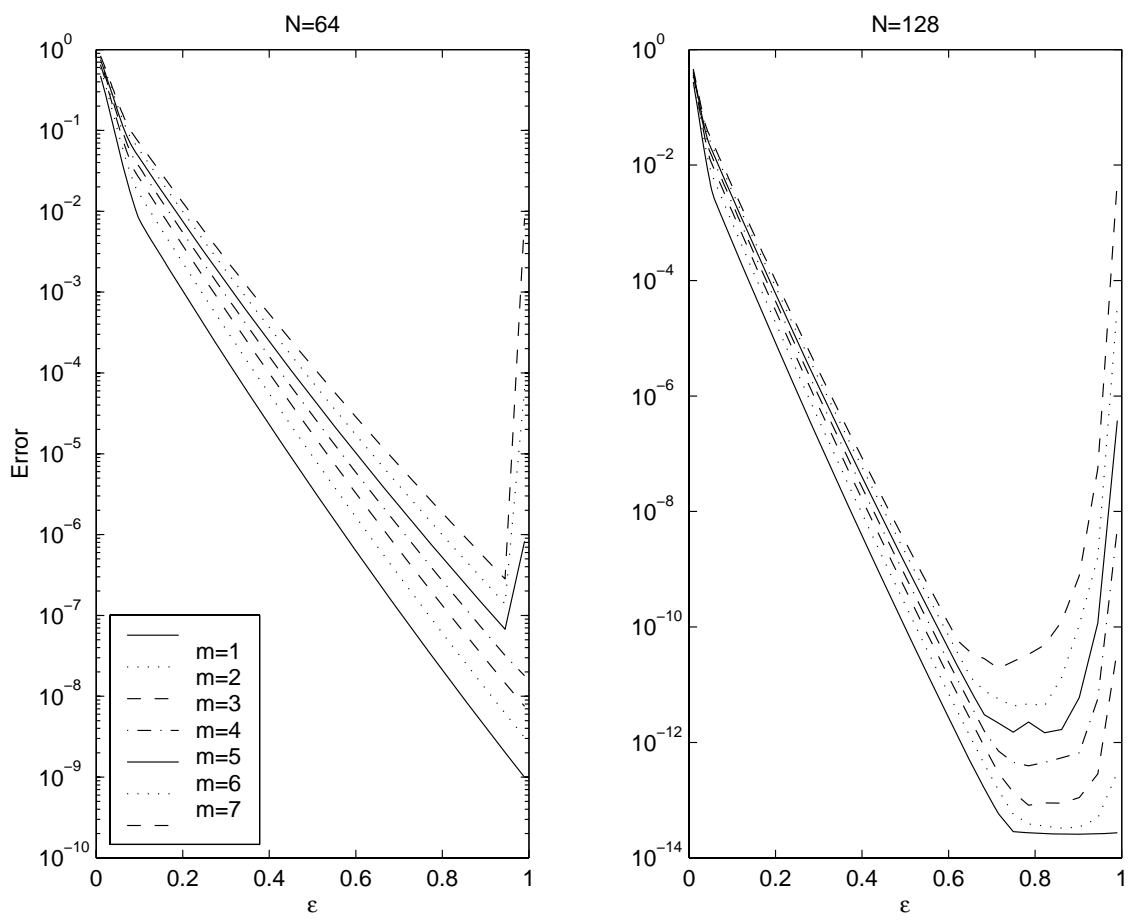

FIG. 2. Semilog-plot of the error versus $\varepsilon$ in Example 1 for different values of $m$ when $f=$ $\varrho_{2}^{m} \mathrm{e}^{\mathrm{i} m \vartheta}$ and $N=64,128$.

Figure 2. In this case we show the dependence of the logarithm of $E$ on $\varepsilon$ for $m=1, \ldots, 7$, for the cases $N=64,128$.

We observe that in both cases, as $m$ increases, the accuracy of the approximation deteriorates. Similar phenomena were observed in previous studies, in particular, in $[13,23,25]$. In [23], in the case of the Dirichlet problem in a disk of radius $\varrho$, with the singularities placed on a circle of radius $R$, it is shown that the error behaves like

$$
E=\mathcal{O}\left(\left(\frac{\varrho}{R}\right)^{N-2 m}\right)
$$

Further, we observe that for large $m$ there is a deterioration of the accuracy for $\varepsilon \approx 1$. This is due to ill-conditioning resulting from the fact that the radius of $S_{R_{2}}$ is close to zero.

Figure 3. We present the dependence of the error on the angular parameters $\alpha$ and $\beta$, when $m=1, \varepsilon=.005, .01, .02, .04, .08, .16$. The two subplots correspond to the cases $N=64$ and $N=128$. For simplicity we took $\alpha=\beta$. Because of symmetry we need to consider only $\alpha \in\left[0, \frac{1}{2}\right]$.

We observe that, for the values of $\varepsilon$ presented, as $\varepsilon$ increases, the error $E$ is optimized for $\alpha \approx \frac{1}{4}$. However, the improvement in the accuracy of the MFS approximation is more visible for smaller values of $\varepsilon$. In particular, for $\varepsilon=.005$, the accuracy of the approximation improves by a factor of ten at $\alpha \approx .1$, which is where the minimum is reached. Further, for larger values of $\varepsilon$ the improvement due to rotation is negligible. These phenomena were also observed in previous studies [20, 21]. 

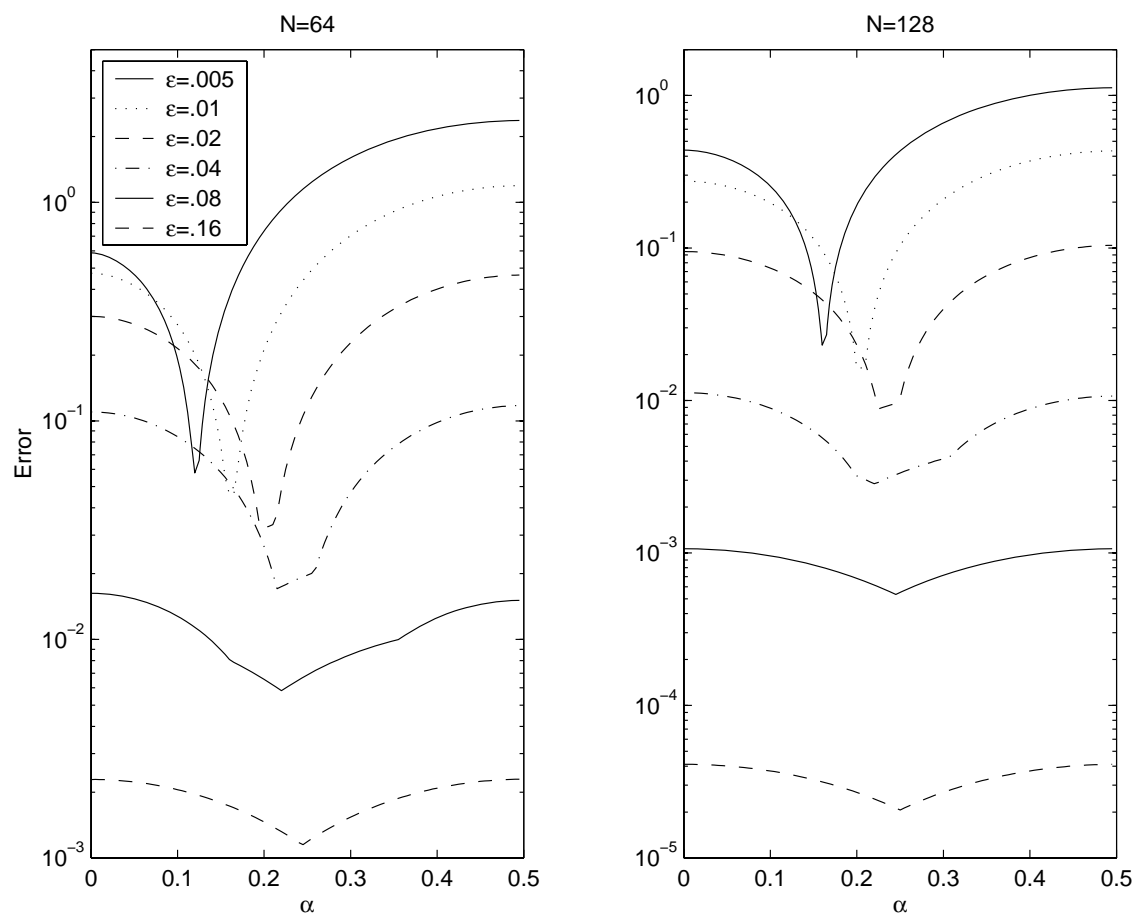

FIG. 3. Semilog-plot of the error versus the angular parameter $\alpha$ when $f=\varrho_{2} e^{\mathrm{i} \vartheta}$ for various values of $\varepsilon$ and $N=64,128$. The parameter $\beta$ is taken to be equal to $\alpha$.

4.2. Three-dimensional case. In this subsection we test numerically the algorithm proposed in section 3 for (3.1e). In the three-dimensional case it is difficult to construct an exact solution for (3.1e), and therefore, in order to test the convergence of the algorithm, we examine, as an estimate of the error, the supremum norm of the differences $u_{i}^{M, N}-u_{i}^{2 M, 2 N}, i=1,2$, for various values of $M$ and $N$. Since these differences are harmonic, from the maximum principle, it is sufficient to consider them on the boundaries $\partial \Omega_{i}, i=1,2$. We therefore calculate

$$
E^{M, N}=\max \left\{E_{1}^{M, N}, E_{2}^{M, N}\right\}
$$

where

$$
\begin{aligned}
& E_{1}^{M, N}=\sup _{x \in \partial \Omega_{1}}\left|u_{1}^{M, N}(\boldsymbol{x})-u_{1}^{2 M, 2 N}(\boldsymbol{x})\right|, \\
& E_{2}^{M, N}=\sup _{x \in \partial \Omega_{1} \cup \partial \Omega_{2}}\left|u_{2}^{M, N}(\boldsymbol{x})-u_{2}^{2 M, 2 N}(\boldsymbol{x})\right| .
\end{aligned}
$$

This quantity was calculated on prescribed grids on the boundaries of the two regions which will be specified for each case.

We consider two examples of boundary data in (3.1c), namely,

Example 1. $f=\cosh (.03 x) \cosh (.04 y) \cos (.05 z)$.

Example 2. $f=\left(9.5-x^{2}-y^{2}-z^{2}\right)^{-1}$.

In all numerical experiments performed in this study, we took $k_{2} / k_{1}=2$. 
4.2.1. Spherical domains. We consider the case where $\Omega_{1}$ and $\Omega_{2}$ are the spheres of radii $\varrho_{1}$ and $\varrho_{2}\left(\varrho_{1}<\varrho_{2}\right)$, respectively, that is,

$$
\Omega_{j}=\left\{(x, y, z) \in \mathbb{R}^{3}: x^{2}+y^{2}+z^{2}<\varrho_{j}^{2}\right\}, \quad j=1,2 .
$$

The singularities $\left\{Q_{\mu, \nu}\right\}_{\mu=1, \nu=1}^{M, N},\left\{S_{\mu, \nu}\right\}_{\mu=1, \nu=1}^{M, N}$, and $\left\{T_{\mu, \nu}\right\}_{\mu=1, \nu=1}^{M, N}$ are placed on the spherical surfaces $\partial \Omega_{Q}, \partial \Omega_{S}$, and $\partial \Omega_{T}$, centered at the origin, of radii $R_{Q}, R_{S}$, and $R_{T}$, respectively, with $R_{S}<\varrho_{1}<R_{T}$ and $\varrho_{2}<R_{Q}$. The coordinates of the singularities are given by (3.3) with

$$
\begin{gathered}
r_{\nu}^{Q}=R_{Q} \sin \vartheta_{\nu}, \quad z_{\nu}^{Q}=R_{Q} \cos \vartheta_{\nu}, \\
r_{\nu}^{S}=R_{S} \sin \vartheta_{\nu}, \quad z_{\nu}^{S}=R_{S} \cos \vartheta_{\nu}, \\
r_{\nu}^{T}=R_{T} \sin \vartheta_{\nu}, \quad z_{\nu}^{T}=R_{T} \cos \vartheta_{\nu},
\end{gathered}
$$

where $\vartheta_{\nu}=\frac{\nu \pi}{N+1}, \nu=1, \ldots, N$. The collocation points $\left\{P_{m, n}^{i}\right\}_{m=1, n=1}^{M, N}$ on the boundaries $\partial \Omega_{i}, i=1,2$, are given by

$$
x_{P_{m, n}^{i}}=\varrho_{i} \sin \vartheta_{n} \cos \varphi_{m}, \quad y_{P_{m, n}}=\varrho_{i} \sin \vartheta_{n} \sin \varphi_{m}, \quad z_{P_{m, n}^{i}}=\varrho_{i} \cos \vartheta_{n} .
$$

Note that we avoid the points corresponding to $\vartheta_{j}=0$ and $\vartheta_{j}=\pi$ as they remain invariant under rotation in the $\varphi$-direction and would lead to singular matrices.

The error was calculated at the points

$$
V_{k, \ell}^{i}=\varrho_{i}\left(\sin \Theta_{\ell} \cos \Phi_{k}, \sin \Theta_{\ell} \sin \Phi_{k}, \cos \Theta_{\ell}\right), \quad i=1,2,
$$

where $\Theta_{\ell}=\frac{\pi \ell}{L+1}$ and $\Phi_{k}=\frac{2 \pi(k-1)}{L}, k, \ell=1, \ldots, L$. In most numerical experiments $L$ was taken to be equal to 31 .

In our numerical experiments, we considered the case of the spheres of radii 1 and 3 , respectively.

Figure 4. We present the logarithm of error, as defined by (4.2c), versus the distance $\varepsilon$ of the three spherical surfaces $\partial \Omega_{Q}, \partial \Omega_{S}$, and $\partial \Omega_{T}$ from the corresponding boundaries $\partial \Omega_{1}, \partial \Omega_{2}$. That is,

$$
R_{Q}=\varrho_{1}+\varepsilon, \quad R_{S}=\varrho_{1}-\varepsilon, \quad R_{T}=\varrho_{2}+\varepsilon .
$$

We consider both Examples 1 and 2 in the unrotated case, i.e., $\alpha=\beta=0$. We took $M=N=12,16,24,32,48$. From this figure we observe that, for both examples, as $\varepsilon$ varies on the interval $\left(10^{-2}, 1\right)$, the error decreases exponentially fast. Also, the rate of convergence is more rapid as $N$ grows.

Figure 5. We present the dependence of the error on the angular parameters $\alpha$ and $\beta$. For simplicity, we considered the case when $\alpha=\beta$. Because of symmetry we need to consider only the case $\alpha \in\left[0, \frac{1}{2}\right]$ and examine the behavior of the error for $M=N=16,24,32,48$ and fixed $\varepsilon$.

We observe that for small $\varepsilon$ an optimal value was reached at about $\alpha \approx \frac{1}{4}$. This behavior can be seen in Figure 5 in the case $\varepsilon=\frac{1}{10}$ for both Examples 1 and 2 . For larger values of $\varepsilon$, the minimum is no longer visible. Similar phenomena have been observed in previous studies [20, 23].

Figure 6. In Figure 6 we present the logarithm of an estimate of the condition number in the $L^{\infty}$-norm of the coefficient matrix in system (3.8) for $m=1$ versus $\varepsilon$, for $N=8,12,16,24,32$, and 48 . As can be seen from this figure, the conditioning of the matrix deteriorates considerably as $\varepsilon$ increases. The estimate of the condition number was calculated using the NAG pair F07ARF-F07AUF. The behavior of the condition number of the matrices in the cases $m=2,3, \ldots M$ is very similar. 

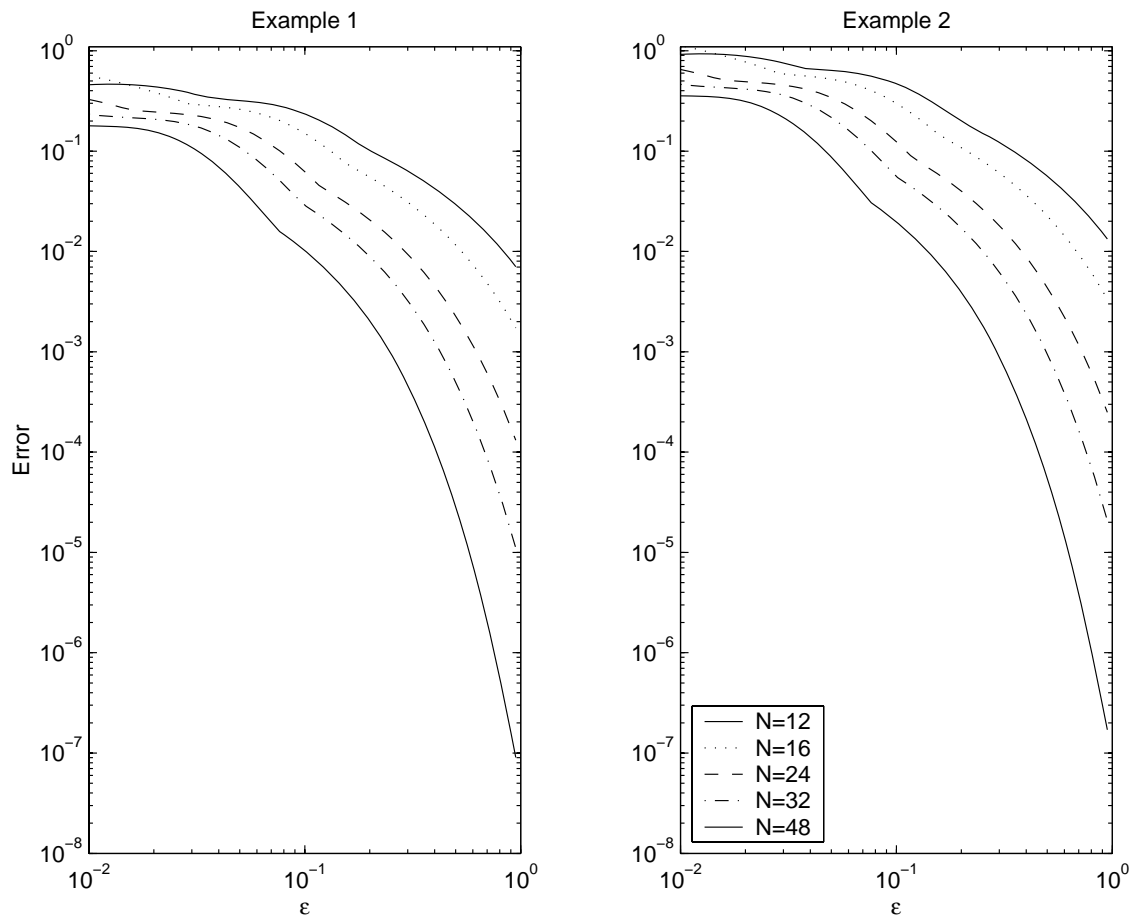

FIG. 4. Loglog-plot of the error versus $\varepsilon$ in Examples 1 and 2 in the case where $\Omega_{1}$ and $\Omega_{2}$ are spheres.
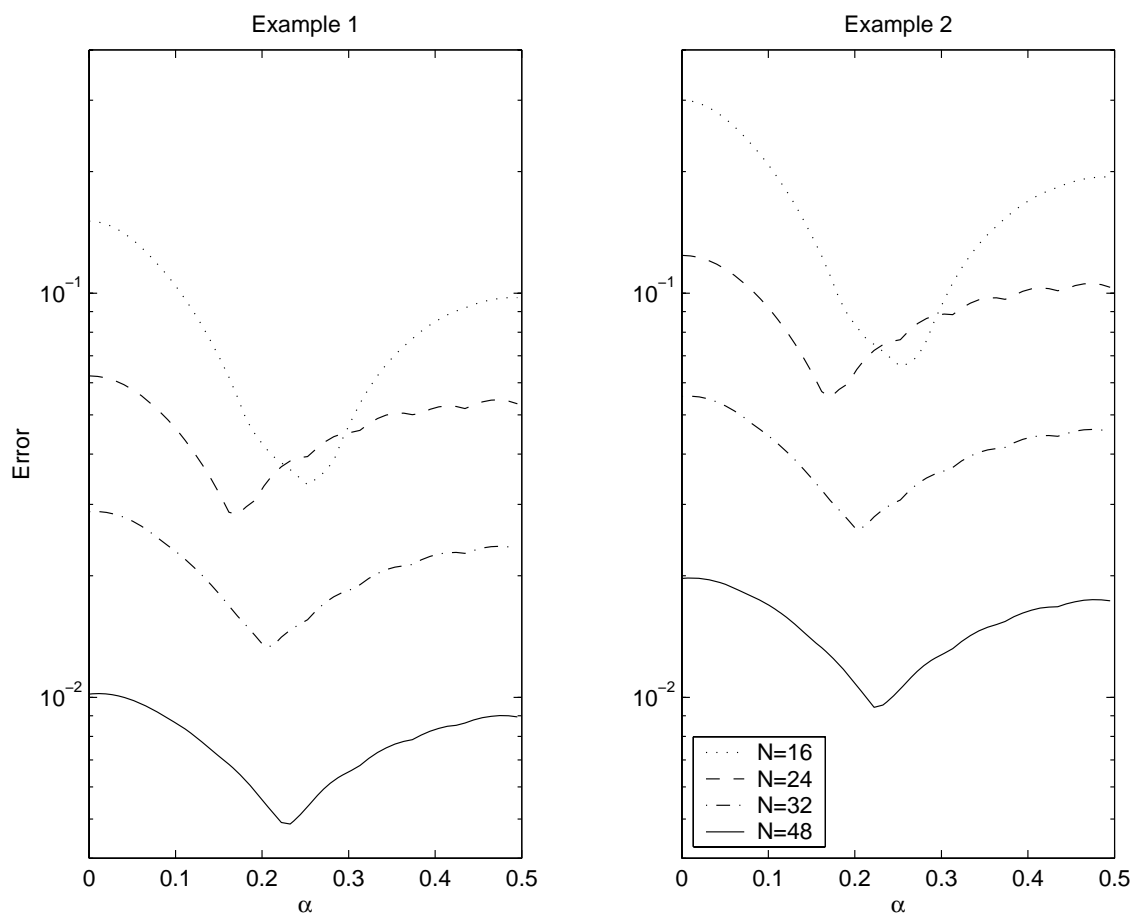

FIG. 5. Spherical domains: Semilog-plot of the error versus the angular parameter $\alpha$ in Examples 1 and 2 for $\varepsilon=1 / 10$. The parameter $\beta$ is taken to be equal to $\alpha$. 


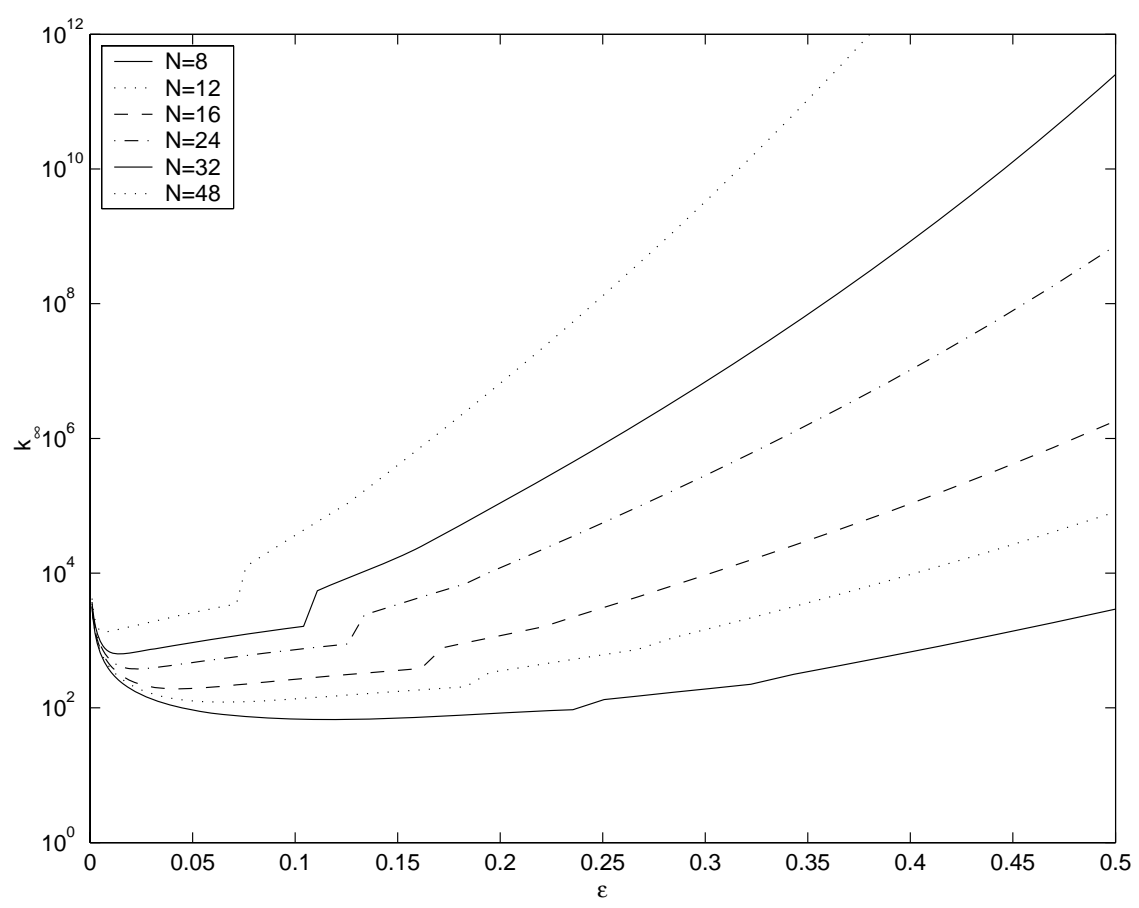

FIG. 6. Spherical domains: Semilog-plot of the condition number $\kappa_{\infty}$ of the matrix in the system (3.8) versus $\varepsilon$.

4.2.2. Cylinder inside a sphere. We consider the case when $\Omega_{1}$ is the cylinder

$$
\Omega_{1}=\left\{(x, y, z) \in \mathbb{R}^{3}: x^{2}+y^{2}<\varrho_{1}^{2},|z|<h\right\}
$$

and $\Omega_{2}$ is the sphere

$$
\Omega_{2}=\left\{(x, y, z) \in \mathbb{R}^{3}: x^{2}+y^{2}+z^{2}<\varrho_{2}^{2}\right\}
$$

where $\varrho_{1}^{2}+h^{2}<\varrho_{2}^{2}$. The boundary points $P_{\mu, \nu}^{2}$ are chosen as in (4.3). The points $P_{\mu, \nu}^{1}$ are given by (3.2), where $\left(r_{n}^{P^{1}}, z_{n}^{P^{1}}\right) \in \mathbb{R}^{2}$ are uniformly distributed on the three sides of the rectangle $\left[0, \varrho_{1}\right] \times[-h, h]$ which is the generator of the cylinder.

The singularities $Q_{\mu, \nu}$ and $S_{\mu, \nu}$ are placed on cylinders similar to $\Omega_{1}$, both at a distance $\varepsilon$ from $\partial \Omega_{1}$. The singularities $T_{\mu, \nu}$ are placed on a sphere of radius $R_{T}$, where $R_{T}-\varrho_{2}=\varepsilon>0$.

In our numerical experiments, we took $h=1, \varrho_{1}=1$, and $\varrho_{2}=3$.

Figure 7. We present the logarithm of the error as defined by (4.2c) versus $\varepsilon$. We apply the method to Example 1 in the unrotated case, and we varied $\varepsilon$ in the interval $\left(10^{-2}, 1\right)$. We took $M=N=8,12,16,24,32,48$. From this figure, we observe that as $\varepsilon$ increases, the error decreases exponentially fast up to about $\varepsilon \approx \frac{1}{2}$. For larger values of $\varepsilon$, the quality of the approximation deteriorates rapidly. For this geometry, the approximation is considerably less accurate than the corresponding approximation in the case of the spherical domains. This is due to the presence of the edges in the cylindrical subdomain. Further, the error in the approximation for this geometry starts deteriorating for smaller values of $\varepsilon$ than for the spherical domains. 


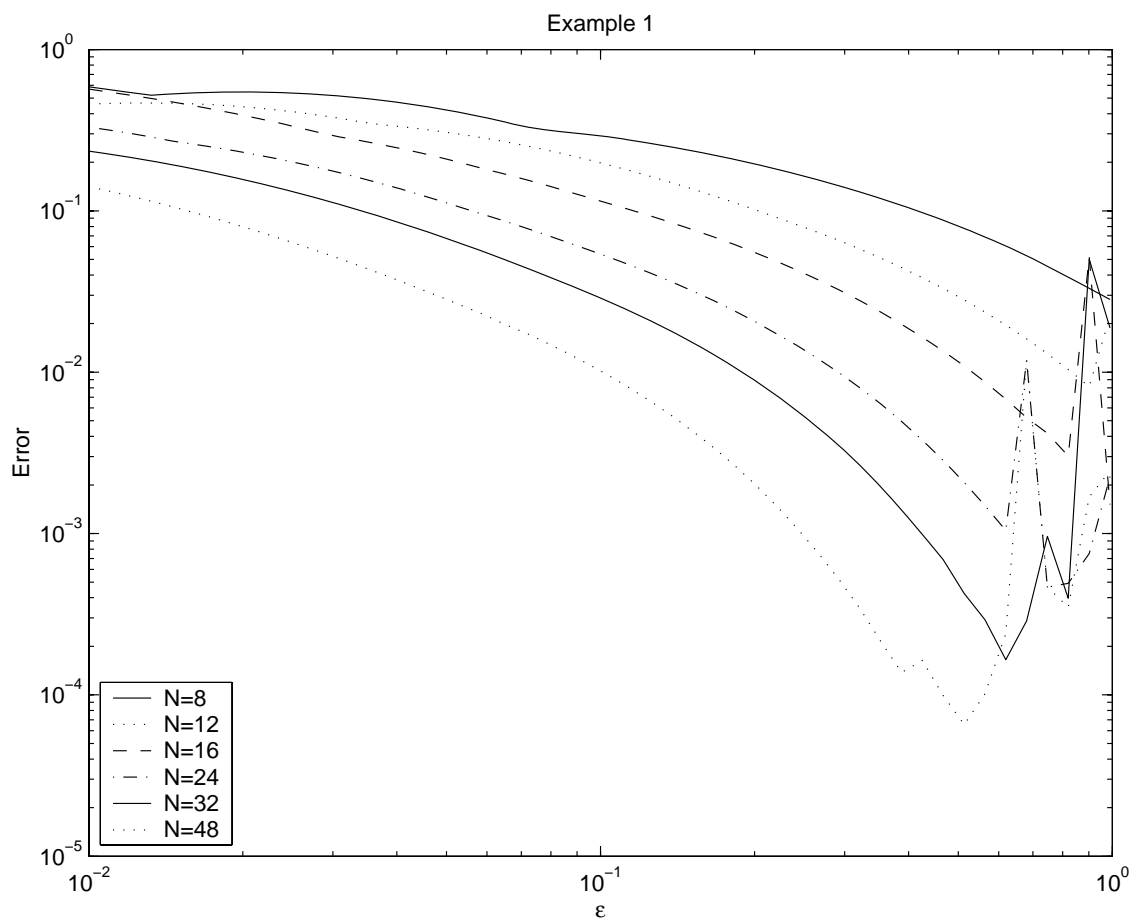

FIG. 7. Cylinder inside a sphere: Loglog-plot of the error versus $\varepsilon$ in Example 1.

Figure 8. We present the dependence of the error on the angular parameters $\alpha$ and $\beta$ in Example 1. For simplicity, again, we considered the case when $\alpha=\beta$, and because of symmetry we examined $\alpha \in\left[0, \frac{1}{2}\right]$. In the first subplot we took $\varepsilon=\frac{1}{10}$ and $M=N=16,24,32,48$. As observed previously, there is a tendency for the optimum to be reached at $\alpha=\frac{1}{4}$ and fixed $\varepsilon$. We observed that for small $\varepsilon$ an optimal value was reached at about $\alpha \approx \frac{1}{4}$.

In the second subplot we present the corresponding results for $\varepsilon=\frac{1}{2}$. As expected, the accuracy is higher, and similar phenomena to those seen in the first subplot are observed.

5. Concluding remarks. In this paper, we apply a meshless boundary collocation method for the solution of certain heat conduction problems in layered materials in two and three dimensions. In two dimensions, we consider materials in concentric regions whereas in three dimensions we consider axisymmetric domains possessing the same axis of symmetry. We employ a domain decomposition technique which leads to systems whose coefficient matrices exhibit block circulant structures. Matrix decomposition methods in conjunction with the use of FFTs enable us to construct efficient algorithms for the numerical solution of these problems.

The algorithms developed in this study could be easily extended to the solution of bimaterial problems in the same geometries in two and three dimensions but governed by different differential equations such as the Helmholtz equation, the biharmonic equation, or the Cauchy-Navier equations of elasticity, in two and three dimensions. Further, this method is not restricted to dealing with bimaterial problems but could also be applied to multilayered materials in corresponding axisymmetric domains in two and three dimensions. 

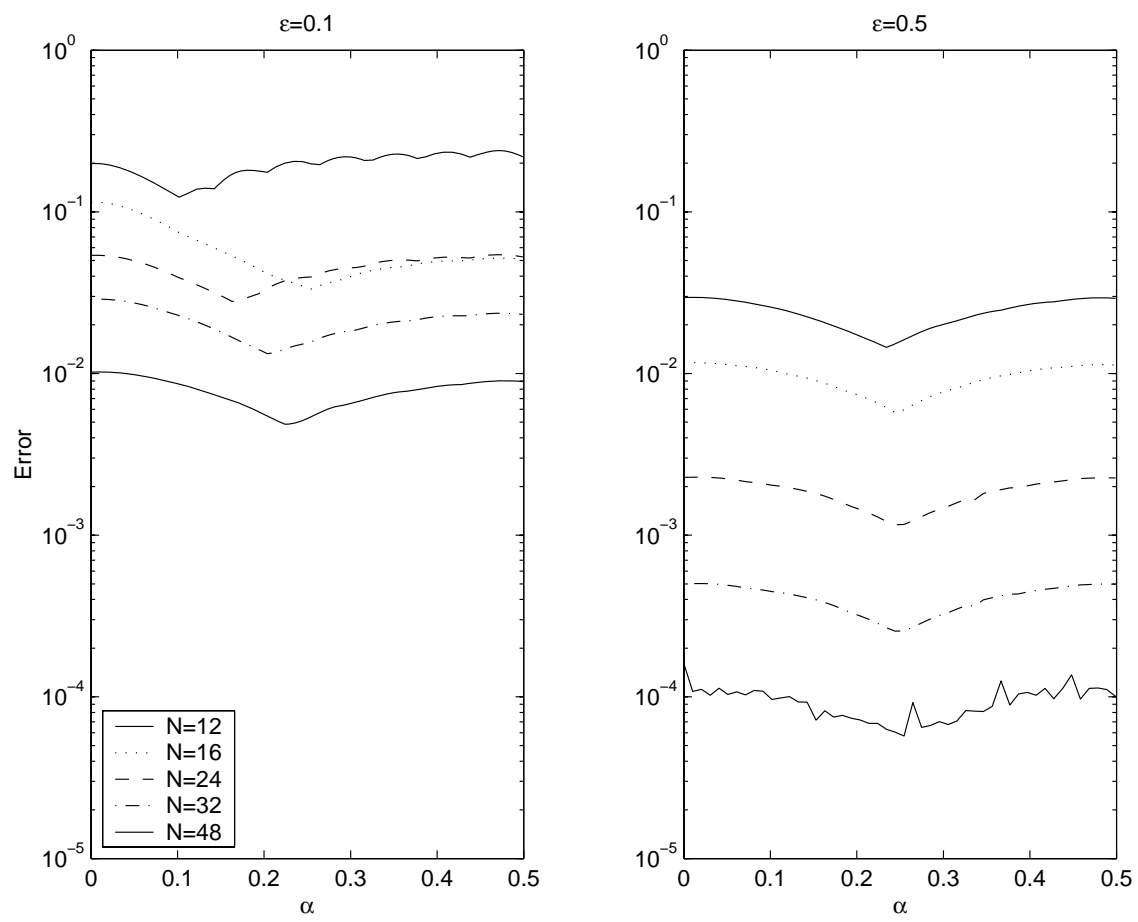

FIG. 8. Cylinder inside a sphere: Semilog-plot of the error versus the angular parameter $\alpha$ for $\varepsilon=1 / 10,1 / 2$. The parameter $\beta$ is taken to be equal to $\alpha$.

Finally, the block circulant structure of the matrices arising in these problems is preserved for other boundary method discretizations. The current algorithm could thus be used when, for example, the BEM is applied to the type of problems examined in this work.

Acknowledgments. The authors wish to thank Professors John R. Berger, Graeme Fairweather, and Paul A. Martin of the Colorado School of Mines for their helpful suggestions.

\section{REFERENCES}

[1] J. R. Berger And A. Karageorghis, The method of fundamental solutions for heat conduction in layered materials, Internat. J. Numer. Methods Engrg., 45 (1999), pp. 1681-1694.

[2] J. R. Berger And A. Karageorghis, The method of fundamental solutions for layered elastic materials, Engrg. Anal. Boundary Elem., 25 (2001), pp. 877-886.

[3] J. T. Chen, J. H. Lin, S. R. Kuo, and S. W. Chyuan, Boundary element analysis for the Helmholtz eigenvalue problems with a multiply connected domain, R. Soc. Lond. Proc. Ser. A Math. Phys. Eng. Sci., 457 (2001), pp. 2521-2546.

[4] H. A. Cho, M. A. Golberg, A. S. Muleshrov, And X. LI, Trefftz methods for time dependent partial differential equations, Comput. Mat. Cont., 1 (2004), pp. 1-37.

[5] P. J. Davis, Circulant Matrices, John Wiley \& Sons, New York, Chichester, Brisbane, 1979.

[6] G. Fairweather and A. Karageorghis, The method of fundamental solutions for elliptic boundary value problems. Numerical treatment of boundary integral equations, Adv. Comput. Math., 9 (1998), pp. 69-95.

[7] G. Fairweather, A. Karageorghis, and P. A. Martin, The method of fundamental solutions for scattering and radiation problems, Engrg. Anal. Boundary Elem., 27 (2003), pp. 759769 . 
[8] G. Fairweather, A. Karageorghis, and Y.-S. Smyrlis, A matrix decomposition MFS algorithm for axisymmetric biharmonic problems, Adv. Comput. Math., 23 (2005), pp. 55-71.

[9] R. T. Fenner, A force superposition approach to plane elastic stress and strain analysis, J. Strain Anal., 36 (2001), pp. 517-529.

[10] M. A. Golberg and C. S. Chen, Discrete Projection Methods for Integral Equations, Computational Mechanics Publications, Southampton, 1997.

[11] M. A. Golberg And C. S. Chen, The method of fundamental solutions for potential, Helmholtz and diffusion problems, in Boundary Integral Methods: Numerical and Mathematical Aspects, Comput. Eng. 1, WIT Press/Computational Mechanics Publications, Boston, MA, 1999, pp. 103-176.

[12] P. Henrici, Fast Fourier methods in computational complex analysis, SIAM Rev., 21 (1979), pp. 481-527.

[13] M. Katsurada, A mathematical study of the charge simulation method. II, J. Fac. Sci. Univ. Tokyo Sect. IA Math., 36 (1989), pp. 135-162.

[14] J. A. Ko€odziej, Applications of the Boundary Collocation Method in Applied Mechanics, Wydawnictwo Politechniki Poznańskiej, Poznań, 2001 (in Polish).

[15] S. R. Kuo, J. T. Chen, And C. X. Huang, Analytical study and numerical experiments for true and spurious eigensolutions of a circular cavity using the real-part dual BEM, Internat. J. Numer. Methods Engrg., 48 (2000), pp. 1401-1422.

[16] U. Lamp, K.-T. Schleicher, And W. L. Wendland, The fast Fourier transform and the numerical solution of one-dimensional boundary integral equations, Numer. Math., 47 (1985), pp. $15-38$.

[17] C. D. Meyer, Matrix Analysis and Applied Linear Algebra, SIAM, Philadelphia, 2000.

[18] M. N. Özışı, Boundary Value Problems of Heat Conduction, International Textbook Company, Scranton, PA, 1968.

[19] J. H. Kane, Boundary Element Analysis in Engineering Continuum Mechanics, Prentice Hall, Engelwood Cliffs, NJ, 1994.

[20] Y.-S. Smyrlis And A. Karageorghis, Some aspects of the method of fundamental solutions for certain harmonic problems, J. Sci. Comput., 16 (2001), pp. 341-371.

[21] Y.-S. Smyrlis And A. Karageorghis, Some aspects of the method of fundamental solutions for certain biharmonic problems, CMES Comput. Model. Eng. Sci., 4 (2003), pp. 535-550.

[22] Y.-S. Smyrlis and A. Karageorghis, A matrix decomposition MFS algorithm for axisymmetric potential problems, Engrg. Anal. Boundary Elem., 28 (2004), pp. 463-474.

[23] Y.-S. Smyrlis And A. KARAgeorghis, Numerical analysis of the MFS for certain harmonic problems, M2AN Math. Model. Numer. Anal., 28 (2004), pp. 495-517.

[24] I. Stakgold, Green's Functions and Boundary Value Problems, John Wiley \& Sons, New York, Chichester, Brisbane, 1979.

[25] T. Tsangaris, Y.-S. Smyrlis, and A. Karageorghis, Numerical analysis of the MFS for harmonic problems in annular domains, Numer. Methods Partial Differential Equations, to appear. 